\title{
Double Intravesical Migration of Intrauterine Devices: Presented With Vesical Stone Formation
}

\author{
Mazen Ahmed Ghanemª, b, d, Essa A. Adawi ${ }^{\mathrm{b}}$, Ashraf A. Ghanem ${ }^{\mathrm{c}}$, Fouad M. Zanaty ${ }^{\mathrm{a}}$
}

\begin{abstract}
We described one case of intravesical migration of a double copper-T intrauterine device (IUCD) associated with formation of stones. The diagnosis was made by ultrasound scan and KUB. In this case, we removed the double IUCDs with calculus endoscopically. Our case is the first report of intravesical migration of double IUCDs with stone formation. A description of the IUCD device history, diagnostic workup, treatment data, and the need for awareness of the importance of post-insertion follow-up and the possibility of intravesical migration are discussed.
\end{abstract}

Keywords: IUCD; Uterine perforation; Vesical stone; Cystoscopy

\section{Introduction}

Intrauterine contraceptive device (IUCD) is a widely used reversible contraceptive method, due to its cost-effectiveness and low complication rates [1]. Serious complications are rare; however perforation of the uterus and intravesical intrauterine device was an infrequent complication $[2,3]$. Once an IUCD has penetrated the bladder, it usually becomes encrusted with calculi and associated with lower urinary tract symptoms [4].

\section{Case Report}

A 50-year-old woman, gravida 4, para 4, was admitted to our clinic with a 3 months history of recurrent urinary tract infection, dysuria, and suprapubic pain. Urinalysis revealed the presence of inflammation, and urine culture demonstrated the presence of Escherichia coli. She was treated with antibiotics, with remission of symptoms. There were no data to support a

\footnotetext{
Manuscript submitted May 21, 2018, accepted June 6, 2018

aDepartment of Urology, Menoufiya University, Shebin El-Kom, Egypt

bDepartment of Urology, Gazan University, Gazan, KSA

${ }^{\mathrm{c} G y n e c o l o g y}$ and Obstetric Department, Mansoura University, Mansoura, Egypt

${ }^{\mathrm{d} C}$ Corresponding Author: Mazen Ahmed Ghanem, Department of Urology, Menoufiya University, Shebin El-Kom, Egypt.

Email: mazenghanem99@yahoo.co.uk
}

doi: https://doi.org/10.14740/wjnu353w tendency of urolithiasis.

The patient's medical history indicated that a copperT IUCD had been inserted in 1999, 3 months following her third normal vaginal delivery. She had unexpectedly conceived again 4 years after insertion and had a normal vaginal delivery without any complication. It was assumed that the IUCD had fallen out and another copper-T IUCD was inserted 3 years after her fourth delivery. As per records both devices were placed correctly in the uterus and the procedures were uncomplicated. Hysterectomy was done in 2011 and it was also assumed that the second IUCD was removed with hysterectomy. Pelvic examination was normal. A plain abdominal radiograph showed the presence in the pelvis of a large stone opacity with dimensions of $3.9 \times 3.4 \mathrm{~cm}$ around the horizontal limbs of both double IUCDs (Fig. 1) which was confirmed by pelvic ultrasonography. Cystoscopy confirmed the presence of two intravesical IUCDs, complicated by stone which was fragmented endoscopically using stone crushing forceps. Both fragmented calculus and IUCDs were removed cystoscopically by grasping forceps (Fig. 2). The patient was discharged 1 day after removing the IUCDs, with uneventful evolution.

\section{Discussion}

One of the major complications of IUCD is perforation of uter-

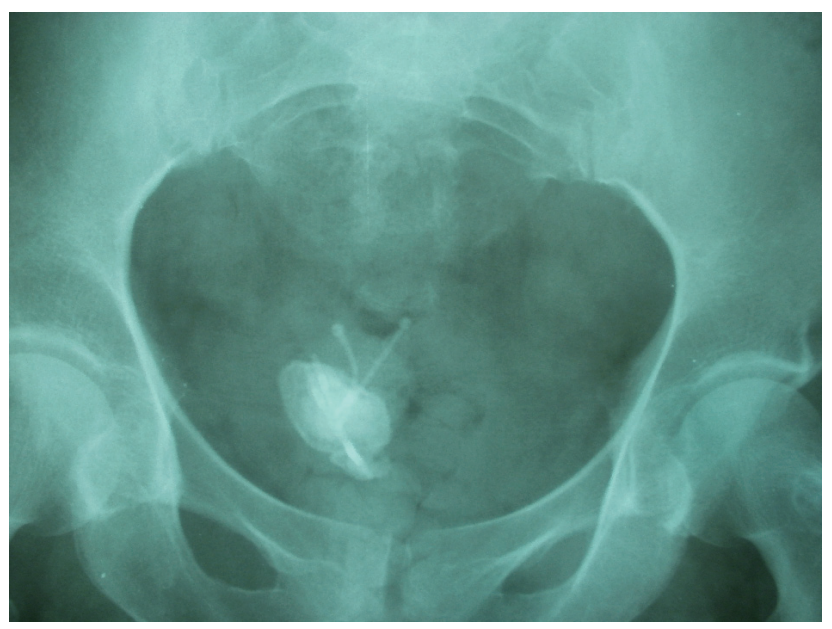

Figure 1. X-ray of the pelvis demonstrates two intrauterine contraceptive devices (IUCDs) within the pelvic cavity with calculus formation on the horizontal limb of both IUCDs. 


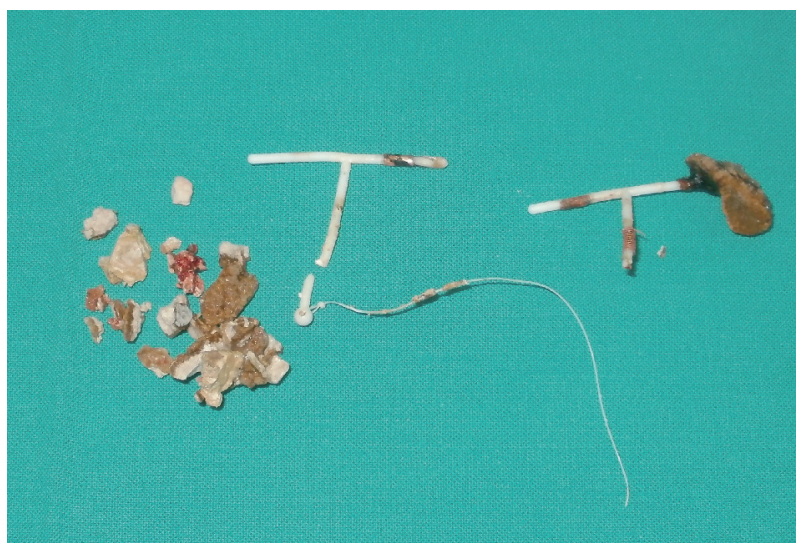

Figure 2. Postoperative image after retrieved two IUCDs and removal of stone endoscopically. Broken copper-T device with the thread can be clearly identified besides fracturing the stone. Another retrieved broken IUCD showing partial calculus formation on one of its horizontal limbs.

ine with overall reported incidence about 0.87 per 1,000 insertions [5]. Furthermore, intravesical migration with secondary bladder stone is yet rarer with incidence about $50 \%$ of the migrated intravesical IUCD [6].

In the present case, two other aspects can be highlighted: this is the first case report of intravesical migration of double IUCD with stone formation. To date, approximately 80 cases of IUCD migration to the bladder have been reported in the scientific literature, and about half of them resulted in stone formation. On review of all reported cases, there was only one intravesical IUCD migration. The second aspect is the long time between the device implantation and the initiation of urinary manifestations 14 years after IUCD insertion suggests that intravesical migration may have occurred in the long term [4]. Also, in the present case, the patient did not receive any imaging study of the urinary tract before she was admitted to our clinics.

However, in such case the exact mechanism that explains uterine perforation and IUCD migrations is not entirely known. Several mechanisms can explain the spontaneous migration of IUCDs, including iatrogenic uterine perforation, spontaneous uterine contraction, involuntary bladder contraction, and peritoneal fluid movement [7]. In our case, we could not ascertain the precise cause of the intrauterine IUCD migrations. Factors increasing the likelihood of uterine perforation include insertion of the device by inexperienced persons, inappropriate positioning and type of the IUCD, susceptible fragile uterine wall because of multiparity and sepsis and the anatomy of the cervix and uterus e.g. extreme posterior uterine position [8].

Presence of IUCD in bladder leads to urinary symptoms and in some cases calculus formation develops over time. They may be asymptomatic or yield abdominal or pelvic signs and symptoms, based on the severity of the problem and location of the IUCD. The degree of stone formation is variable and independent of the duration in bladder. The interval between insertion and symptoms varies from 6 months to 16 years. All IUCDs are radio-opaque; therefore, plain pelvic radiography may be used for detection of the IUCD. The plain film diag- noses a bladder perforation by demonstrating a bladder stone with an attached IUCD that has served as a nidus for the deposition of radiopaque urinary salts [9].

Ultrasonography is a sensitive diagnostic tool of bladder diseases and in cases of loss of the IUCD [6]. However, in cases of partial migrations, further diagnostic methods may be required. Computerized tomography is very effective in demonstrating the IUCD relations with adjacent structures and allows us to evaluate other possible causes of lower UT dysfunction symptoms [1].

Although there is controversy regarding the management of extrauterine IUCDs in asymptomatic patients, there appears to be a consensus that all extrauterine copper-laden devices should be removed as soon as possible after establishment of the diagnosis, regardless of its type and location, as copper IUCDs result in inflammatory reactions and adhesion $[10,11]$. Treatment options for IUCDs that migrate into the bladder vary. Cystoscopic extraction of the device and stones is the preferred approach in the removal of intravesical foreign bodies that are located completely inside the bladder, or for IUCDs that exhibit formation of small calculi. Endourological management is the preferred approach due to high success rate and less morbidity. Lithotripsy of bladder stones may be required preceding the extractions in large stones [12].

In conclusion, chronic pelvic pain and dysfunctional voiding symptoms with a history of an unretrieved IUCD must be carefully researched for possible perforation of the uterus and intravesical migration of IUCD. Also, when IUCD strings are not observed in the vagina during examination and are not detected in the endometrial cavity using ultrasonography, a physician must consider IUCD displacement and warrants investigation including urinary tract imaging study. An IUCD that migrates into the bladder must be removed because of associated complications. Finally, cystoscopy is the optimal approach in evaluating intravesical calcifications and in the case of diagnosis already defined by imaging methods; endoscopy is performed with therapeutic intention.

\section{Funding Support}

This article was not funded by industry nor had any connections with industry. Also, we did not receive any funding to conduct the study.

\section{Conflict of Interest}

None declared.

\section{References}

1. El-Hefnawy AS, El-Nahas AR, Osman Y, Bazeed MA. Urinary complications of migrated intrauterine contraceptive device. Int Urogynecol J Pelvic Floor Dysfunct. 2008;19(2):241-245.

2. Sano M, Nemoto K, Miura T, Suzuki Y. Endoscopic 
Treatment of Intrauterine Device Migration into the Bladder with Stone Formation. J Endourol Case Rep. 2017;3(1):105-107.

3. Shen JK, Ko EY, Staack A. Early pregnancy likely caused by an intravesical intrauterine device. Can J Urol. 2016;23(5):8487-8490.

4. Ozgur A, Sismanoglu A, Yazici C, Cosar E, Tezen D, Ilker Y. Intravesical stone formation on intrauterine contraceptive device. Int Urol Nephrol. 2004;36(3):345-348.

5. Tuncay YA, Tuncay E, Guzin K, Ozturk D, Omurcan C, Yucel N. Transuterine migration as a complication of intrauterine contraceptive devices: six case reports. Eur J Contracept Reprod Health Care. 2004;9(3):194200.

6. Sataa S, Sami B, Sabeur R, Karim C, Ali H. Bladder calculus resulting from the migration of an intrauterine contraceptive device: a report of ten cases. Int J Nephrol Urol. 2011;3(1):54-61.

7. Bera MK, Das CC. Migration of intrauterine copper 7 leading to vesical calculus. Indian J Surg. 2009;71(5):279281.

8. Singh I. Intravesical $\mathrm{Cu}-\mathrm{T}$ emigration: an atypical and infrequent cause of vesical calculus. Int Urol Nephrol. 2007;39(2):457-459.

9. Gunbey HP, Sayit AT, Idilman IS, Aksoy O. Migration of intrauterine devices with radiological findings: report on two cases. BMJ Case Rep. 2014;2014.

10. Markovitch O, Klein Z, Gidoni Y, Holzinger M, Beyth Y. Extrauterine mislocated IUD: is surgical removal mandatory? Contraception. 2002;66(2):105-108.

11. De Silva WSL, Kodithuwakku K, Aponsu GUE, Rathnayake RMM, Rajasegaram E. A large bladder stone caused by the intravesical migration of an intrauterine contraceptive device: a case report. J Med Case Rep. 2017;11(1):293.

12. Tosun M, Celik H, Yavuz E, Cetinkaya MB. Intravesical migration of an intrauterine device detected in a pregnant woman. Can Urol Assoc J. 2010;4(5):E141-143. 